\title{
On the temperature distribution in liquid-vapor phase change between plane liquid surfaces
}

\author{
P. N. Shankarand M.D. Deshpande \\ National Aeronautical Laboratory, Bangalore 560 017, India
}

(Received 23 May 1989; accepted 10 January 1990)

\begin{abstract}
The problem of temperature distribution in liquid-vapor phase change between plane liquid surfaces is considered both analytically and experimentally. Classical kinetic theory calculations indicate that for a monatomic vapor, large temperature jumps exist at the interfaces, large enough possibly to cause the temperature distribution to be anomalous. Surprisingly, no experimental results have been reported so far. A continuum analysis, with special consideration of the Knudsen layers, clearly establishes the adverse roles played by polyatomic molecules and the presence of a contaminant gas. The need to work with monatomic vapors under low levels of contamination is clearly indicated. An experimental setup was constructed to measure temperature distributions in the vapor between an evaporating liquid surface and a cooler condensing surface. Temperature distributions were measured using Chromel-Alumel thermocouples. Measurements were made in water, Freon 113 , and mercury. The temperature profiles obtained in mercury, over a wide range of temperatures, clearly show large temperature jumps at the interfaces, as large as almost $50 \%$ of the applied temperature difference. These results appear to be the first to confirm this important phenomenon.
\end{abstract}

\section{INTRODUCTION}

An important unresolved issue in contemporary fluid mechanics has been the question of the temperature distribution in liquid-vapor phase change between plane liquid surfaces. Consider a pure vapor A confined between two infinite parallel surfaces of its own condensed phase that are at temperatures $\widehat{T}_{0}$ and $\hat{T}_{1}$, respectively (see Fig. 1). If $\widehat{T}_{0}>\widehat{T}_{1}$ liquid will evaporate from the lower surface and condense on the top surface. It was $\mathrm{Pao}^{1}$ who first pointed out from a kinetic theory analysis that for a monatomic vapor the temperature would suffer large jumps at the interfaces; large enough, indeed, for the temperature gradient in the bulk of the vapor to possibly oppose the applied gradient. These remarkable results hold even in the continuum limit, though contamination by an inert gas and molecular internal energy can drastically reduce the effects. ${ }^{2}$

These results, though theoretically confirmed by many others, ${ }^{3,4}$ have led to much controversy over the past 19 years with many suggestions that experiments be done to settle the issue. Serious doubts have been raised about the formulation and boundary conditions and even about whether the results violate basic physical laws. ${ }^{5}$ The concern about this issue was sufficient that Hermans and Beenakker ${ }^{6}$ have tried to show that the inverted temperature profile cannot be excluded on the basis of nonequilibrium thermodynamics. The strange phenomena persist for most reasonable modifications of the boundary conditions (Cercignani et $\mathrm{fl} /{ }^{7}$ ).A surprising fact is that in spite of all the concern no experimental results, to our knowledge, have been reported on this problem. The present investigation was undertaken to fill this important gap.

For the idealized situation shown in Fig. 1, most of the initial theoretical work assumed that the vapor was pure, i.e., uncontaminated, and that it was monatomic. Under these circumstances the theory predicts (see, for example, Aoki and Cercignani $\left.{ }^{4}\right)$ that in the continuum limit, the mass flux $\mathrm{m}$, and the temperature distribution $T(y)$ in the vapor are approximately given by

$$
\begin{aligned}
& m=(4 / 9) \pi \rho_{\mathrm{A}}^{*} \sqrt{2 \pi R_{\mathrm{A}} T^{*}}\left(\hat{p}_{\mathrm{A} 0}^{\prime}-\hat{p}_{\mathrm{A} 1}^{\prime}\right), \\
& T(y)=T_{0}+\left[\left(T_{1}-T_{0}\right) /\left(e^{H / \delta}-1\right)\right]\left(e^{y / \delta}-1\right), \\
& \hat{T}_{0}-T_{0}=T_{1}-\hat{T}_{\mathrm{T}}=\frac{1}{9}\left(\frac{T^{*}}{p_{\mathrm{A}}^{*}} \frac{d p_{\mathrm{A}}^{*}}{d T^{*}}\right)\left(\hat{T}_{0}-\hat{T}_{1}\right) \\
& \quad=\frac{(\beta+1)}{9} \Delta \mathrm{r}, \\
& \delta=k_{\mathrm{A}} / \dot{m} C_{p_{\mathrm{A}}}, \quad \\
& (\beta+1)=\left(\frac{T^{*}}{p_{\mathrm{A}}^{*}} \frac{d p_{\mathrm{A}}^{*}}{d T^{*}}\right) \approx \frac{L}{R_{\mathrm{A}} T^{*}} .
\end{aligned}
$$

In the above, quantities with asterisks refer to the equilibrium conditions, primes refer to nondimensional perturbations, carets refer to liquid conditions, and $T_{0}$ and $T_{1}$ are the vapor temperatures at the lower and upper surfaces, respectively. Note that $S$ is a thickness based on the mass flux and the thermal properties of the vapor, ( 3 is a fluid parameter related to the latent heat, and the subscript A refers to

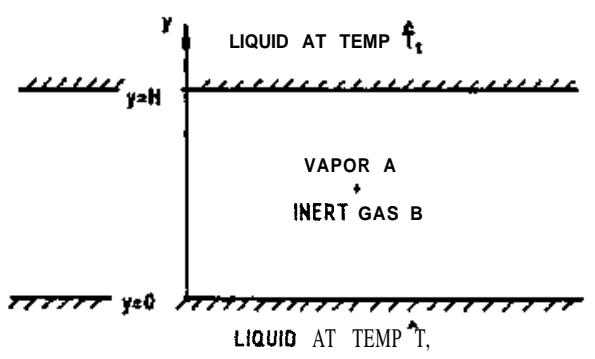

FIG. 1. Evaporation and condensation between plane liquid surfaces. 
the pure vapor A. The temperature jumps are clearly indicated by (3) and a little thought shows that if TO $-T_{0}>\frac{1}{2}\left(T_{0}-T_{1}\right)$ the temperature gradient in the vapor opposes the applied gradient. The equations also show that if the mass flux is sufficiently high the temperature in the fluid is approximately constant at the value $T_{0}$ and jumps to the value $T_{1}$ at $y=H$ over a distance (often small) of the order $S$. It may be noted here that Plesset ${ }^{8}$ derived the form (2) for the temperature distribution but did not account for the jumps (3).

In any attempt to observe experimentally the phenomenon, a number of issues have to be considered, principal among them being the role of contamination and the effects of polyatomic molecules. One might naively ignore these factors but analysis shows these effects to be severely adverse, i.e., a low $\gamma_{\mathrm{A}}\left(=C_{P_{\mathrm{A}}} / C_{V_{\mathrm{A}}}\right)$ and contamination (usually air) severely reduce the temperatures jumps. Thus (1) and (3) become

$$
\begin{aligned}
& m=\bar{K}_{1} \dot{M}, \\
& \hat{T}_{0}-T_{0}=K_{2}(\beta+1)\left(\hat{T}_{0}-\hat{T}_{1}\right)=T_{1}-\hat{T}_{1},
\end{aligned}
$$

where $\dot{M}$ is the mass flux for an uncontaminated monatomic vapor given by ( 1 ) and $\bar{K}_{1}$ and $K_{2}$ are correction factors. Here, $\bar{K}_{1}$ is drastically reduced by the presence of an inert gas $\mathrm{B}$, while $K_{2}$ decreases rapidly both as $\gamma_{\mathrm{A}} \rightarrow 1$ and as $p_{\mathrm{B}}^{*}$ increases. These results have a very serious bearing on any experimental verification. In fact, we believe that our success in observing large temperature jumps at the boundaries was due to a proper recognition of these factors and designing the experiments appropriately.

In Sec. II we outline a continuum analysis of the effects of real gas properties and contamination, in Secs. III and IV the experimental apparatus and procedures are described, and the results and conclusions are discussed in Secs. V and VI, respectively.

\section{AN ANALYSIS OF THE EFFECTS OF REAL GAS PROPERTIES AND CONTAMINATION}

The analysis to be outlined here was specifically carried out to estimate the effects of real gas properties and contamination that will invariably be present in an actual experimental situation. Thus in Fig. 1 gas A need not be monatomic and an inert contaminant gas B is present. We are interested in the effects on the temperature distribution of gas properties such as specific heat $C_{P \mathrm{~A}}$, the ratio of the specific heats $\gamma_{\mathrm{A}}$, etc., and also of the binary diffusion coefficient $D_{\mathrm{AB}}$. It may be noted that kinetic theory treatments of the gas mixture case for monatomic gases and the effects of internal degrees of freedom have been given by Onishi ${ }^{9}$ and Cercignani et al., ${ }^{7}$ respectively. However, these independent treatments do not, in our opinion, stress the practical implications of the combined effects. The continuum analysis, following Marble's analysis for the pure vapor case, is simpler and clearly emphasizes the combined roles of real gas properties and contamination.

\section{A. Outline of analysis}

The analysis proceeds by solving the continuum gasdynamic equations in the bulk of the gas mixture with initially unknown

The

layers, where the distribution functions are . two are analyzed separately; the boundary for the continuum analysis, which can be completed.

In outline form, the is steps.

by the following

(1) The

$\operatorname{arc}$

solved for the vapor-gas mixture in the bulk of the flujd using the binary diffusion coeficient $D_{A}$ to account for the diffusion process,

(2) The Knudsen layers are analyzed separately assuming two-sided Maxwellian distributions ( see Fig, 2) for both species and ensuring that mass, momentum,

conserved and equal to their continuum values.

(3) In order to account, in the Knudsen layers, for the contributions to the internal energy from molecular internal degrees of freedom we assume the following. For molecules leaving the liquid surface the effective temperature, for this purpose, is assumed to be the liquid temperature. We know that for molecules scattered into the liquid the effective temperature must lie (at the lower surface as shown in Fig. 2) between $T_{0}$ and $T_{2}$, where $T_{0}=\frac{1}{2}\left(\hat{T}_{0}+T_{2}\right)$ is the vaportemperature at the lower interface. We therefore that these molecules have a temperature (for purposes of computing the nontransiational internal energy) given by

$$
(\lambda / 2) \hat{T}_{0}+[1-(\lambda / 2)] T_{2}, \quad 0 \leqslant \lambda \leqslant 1 \text {. }
$$

The results are not too sensitive to the actual value of $\mathrm{I}$.

(4) The equations are then linearized. their equilibrium values and solved.

All the details of the above are _ in Shankar. ${ }^{2}$

\section{B. The mass fluxand temperature jumps}

The expressions for the mass flux and temperature jumps can be simplified by introducing the following notation:

$$
\begin{aligned}
& C_{V \mathrm{~A}}^{i}-C_{V \mathrm{~A}}-\frac{3}{2} R_{\mathrm{A}}, \\
& \bar{C}_{\mathrm{r}}-C_{\mathrm{T}}-C_{\mathrm{r}}^{i}-C_{\mathrm{F}}\left(C_{\mathrm{r}}-\frac{3}{2} R_{\mathrm{n}},\right. \\
& \phi=\frac{1}{R_{\mathrm{A}}}\left(1-\frac{\lambda}{2}\right)\left(C_{V \mathrm{~A}}^{i}+\frac{p_{\mathrm{B}}^{*}}{p_{\mathrm{A}}^{*}} \sqrt{\frac{R_{\mathrm{A}}}{R_{\mathrm{B}}}} C_{V \mathrm{~B}}^{i}\right),
\end{aligned}
$$

where quantities, without subscripts refer to the mixture and subscripts A and B refer to the vapor and inert gas, respectively.

The mass flux and temperature jumps are then given by

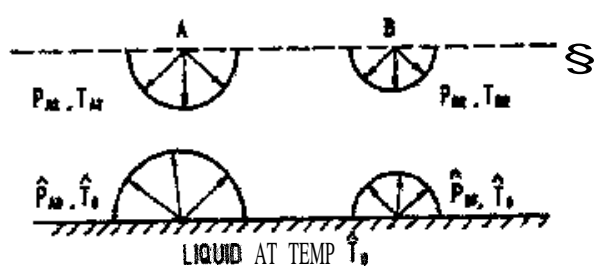

FIG. 2. The two-sided distributions at the tower interfate. 


$$
\begin{aligned}
\dot{m}=\dot{m}_{\mathrm{A}}= & 2\left(1+\frac{\phi}{2}+\frac{p_{\mathrm{B}}^{*}}{p_{\mathrm{A}}^{*}} \sqrt{\frac{R_{\mathrm{B}}}{R_{\mathrm{A}}}}\right)\left(p_{\mathrm{A}}^{*} \sqrt{2 \pi R_{\mathrm{A}} T^{*}}\right) \\
& \times\left(\hat{p}_{\mathrm{A} 0}^{\prime}-\hat{p}_{\mathrm{A} 1}^{\prime}\right)\left[\left(1+\phi+2 \frac{p_{\mathrm{B}}^{*}}{p_{\mathrm{A}}^{*}} \sqrt{\frac{R_{\mathrm{B}}}{R_{\mathrm{A}}}}\right)\right. \\
& +\underset{2 R_{\mathrm{A}}}{\bar{C}_{P}}+\left(1+\frac{\phi}{2}+\frac{p_{\mathrm{B}}^{*}}{p_{\mathrm{A}}^{*}} \sqrt{\frac{R_{\mathrm{B}}}{R_{\mathrm{A}}}}\right) \\
& \times\left(\begin{array}{c}
2 H \\
\left(\rho^{*} D_{\mathrm{AB}}\right) \sqrt{2 \pi R_{\mathrm{A}} T^{*}}
\end{array}\right]^{-1},
\end{aligned}
$$

$$
\begin{aligned}
\hat{I}_{0}-1 \sigma= & \frac{T^{*}}{2} \frac{\left[\left(\bar{C}_{\rho} / 2 R_{\mathrm{A}}\right)-1\right]}{\left[1+(\phi / 2)+\left(p_{\mathrm{B}}^{*} / p_{\mathrm{A}}^{*}\right) \sqrt{R_{\mathrm{B}} / R_{\mathrm{A}}}\right]} \\
& x \frac{\sqrt{2 \pi R_{\mathrm{A}} T^{*}}}{p_{\mathrm{A}}^{*}} \dot{m} \\
= & T_{1}-\widehat{T}_{1},
\end{aligned}
$$

where the quantities with asterisks are equilibrium values and $\hat{p}_{\mathrm{A} 0}^{\prime}$ and $\hat{p}_{\mathrm{A} 1}^{\prime}$ are the dimensionless vapor pressures at the lower and upper surfaces, respectively.These expressions contain the effects of interest to us, i.e., those of real gas properties and contamination. Note that (13) can also be written in the form

$\widehat{T}_{0}-T_{0}=K_{2}(\beta+1)\left(\hat{T}_{0}-\hat{T}_{1}\right)=K_{2}(\beta+1) \Delta \hat{T}$,

where

$$
\begin{aligned}
K_{2}= & \left(\frac{\bar{C}_{p}}{2 R_{\mathrm{A}}}-1\right)\left[\left(1+\phi+2 \frac{p_{\mathrm{B}}^{*}}{p_{A}^{*}} \sqrt{\frac{R_{\mathrm{B}}}{R_{\mathrm{A}}}}\right)\right. \\
& +\frac{\bar{C}_{p}}{2 R_{\mathrm{A}}}+\left(1+\frac{\phi}{2}+\frac{p_{\mathrm{B}}^{*}}{p_{\mathrm{A}}^{*}} \sqrt{\frac{R_{\mathrm{B}}}{R_{\mathrm{A}}}}\right) \\
& \left.\times\left(\frac{2 H}{\rho^{*} D_{\mathrm{AB}}}\right) \frac{p_{\mathrm{B}}^{*}}{\sqrt{2 \pi R_{\mathrm{A}} T^{*}}}\right]^{-1} .
\end{aligned}
$$

When no inert gas is present, i.e., when $p_{\mathrm{B}}^{*}=0$ these expressions simplify to

$$
\begin{aligned}
\dot{m} & =K_{1}(\gamma, \lambda) \dot{M} \\
& =K_{1}(\gamma, \lambda)\left[(4 / 9 \pi) \rho_{\mathrm{A}}^{*} \sqrt{2 \pi R_{\mathrm{A}} T^{*}} \Delta \hat{p}_{\mathrm{A}}^{\prime}\right], \\
\widehat{T}_{0} & -T_{0}=[(1+\beta) / 9] F(\gamma, \lambda) \Delta \bar{T},
\end{aligned}
$$

showing the effects of molecular internal structure alone. Figure 3 shows the relative insensitivity of $K_{1}$ and $F$ to the value of $A$. More important, although the mass flux is hardly affected by changes in 7 , the temperature jump is drastically reduced by reductions in 7 and in fact vanishes for $7=1$. This agrees qualitatively with the results of Cercignani et al. ${ }^{7}$ Note that for $\gamma=1.3$ the temperature jump is about $40 \%$ less than the monatomic gas value.

The effect of the contaminant is clearly to reduce the mass flux and the jumps and calculations show that the effects are usually pronounced. The diffusion term $\left[H\left(2 \pi R_{\mathrm{A}} T^{*}\right)^{1 / 2} / \pi D_{\mathrm{AB}}\right]$ plays a dominant role in the effect of contamination.

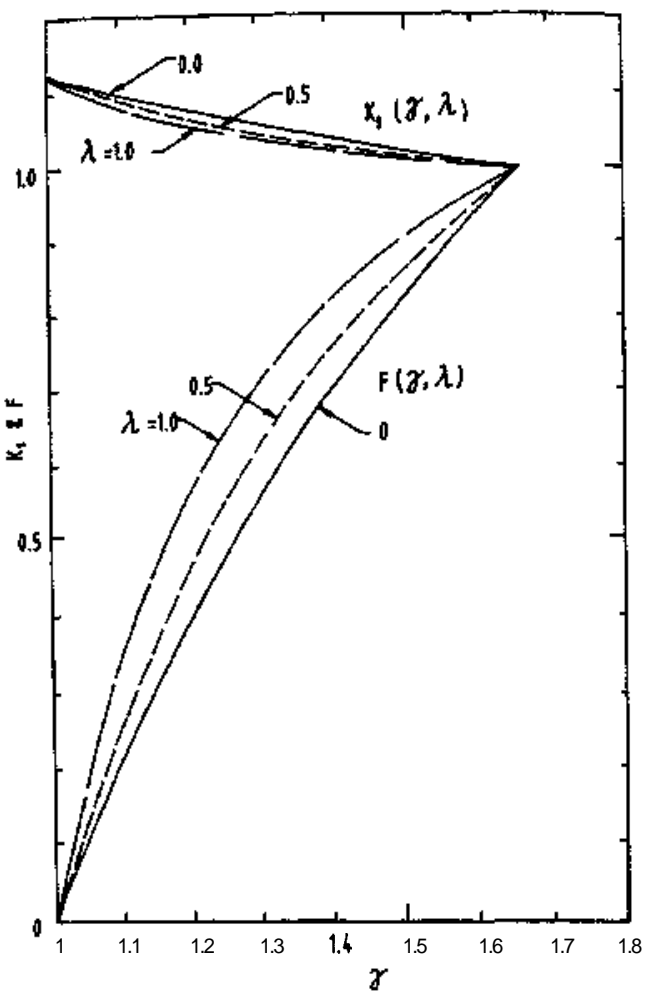

FIG. 3. The mass flux and temperature parameters $K_{1}(\gamma, \lambda)$ and $F(\gamma, \lambda)$.

Finally, the shape of the temperature distribution curve is to be noted. The distribution (2) is exponential in character with jumps at the boundaries as given by (14) and (15).

Note that only if

$$
K_{2}(\beta+1)>\text {. }
$$

will the temperature gradient in the fluid be anomalous, i.e., the temperature will decrease from the cold liquid surface to the hot liquid surface. When the parameter equals 0.5 , the temperature is constant across the gap. Most fluids, far from the boiling point, have values of $(\beta+1)$ between 15 and 20 . Table I shows how the critical value $\gamma_{C}$ of 7 , below which the temperature inversion is absent (for the pure vapor), depends on $(\beta+1)$ and $\lambda$. Note the mild sensitivity on $\lambda$. Table I also shows the values of $\gamma_{c}$ computed from formula (8) of Cercignani et al? assuming that $7=(j+5) /(j+3)$. The excellent agreement with our results gives us confidence that the results are not sensitive to the details of the model chosen. The table clearly shows that it is unlikely that the inversion will be observable in vapors whose value of 7 is less than about 1.1. Another important fact to be noted is that when $\delta \ll H$, for example, when $m$ is high, the temperature is almost uniform across the gap at the value equal to the hot

TABLE I. The critical specific heat ratio below which temperature inversion is absent for a pure vapor.

\begin{tabular}{cccc}
\hline \hline$(1+\beta)$ & $\gamma_{c}(\lambda=0)$ & $\gamma_{c}(\lambda=1)$ & $\gamma_{c}$ (Cercignani et al. $)$ \\
\hline 15.0 & 1.148 & 1.083 & 1.167 \\
17.5 & 1.125 & 1.069 & 1.141 \\
20.0 & 1.108 & 1.059 & 1.122 \\
\hline
\end{tabular}


wall value $T_{0}$ with an abrupt rise or decrease to the cold wall value $T_{1}$ near $y=H$. Thus the gradient will be observable only if 8 is sufficiently large, i.e., $m$ will have to be small.

Figures 4 and 5 show the results of typical calculations for the practically important cases of water vapor and mercury, respectively, contaminated in each case with air. In Fig. 4 when $p_{\mathrm{B}}^{*}$ is small the temperature distribution is anomalous but for practical purposes uniform at the value $T_{0}$ with an abrupt jump near $y=H$. This is because of the high mass flux $\mathrm{m}$. On the other hand, with just about $1 \%$ air, diffusion effects are dominant. Then, the temperature distribution is normal,the jumps are small, and the gradients are small everywhere because $m$ is small (i.e., 3 is large).

For mercury vapor, shown in Fig. 5, the results are significantly different. The anomalous gradients are more easily observable in this case. Since the vapor pressure is lower, the mass flux is greatly reduced causing $5\left(=k / \dot{m} C_{p}\right)$ to be larger. In this case the benefits of $y-\frac{5}{3}$ are also obtained. The effects of contamination are also reduced: even with $1 \%$ contamination the anomalous gradient persists.

\section{Practical implications}

We conclude by listing a few practical implications of the theory as regards its experimental confirmation.

(1) Since real gas effects so seriously and adversely affect the temperature jumps, it is best to work with monatomic and diatomic molecules, e.g., mercury and iodine.

(2) In order to be able to observe the inverse gradient in the vapor $8=k / \dot{m} C_{p}$ has to be large. This must be done by lowering the mass flux. For reasonable applied temperature differences, this means that one must work at low vapor pressures, probably around 0.1 to 1 Torr.

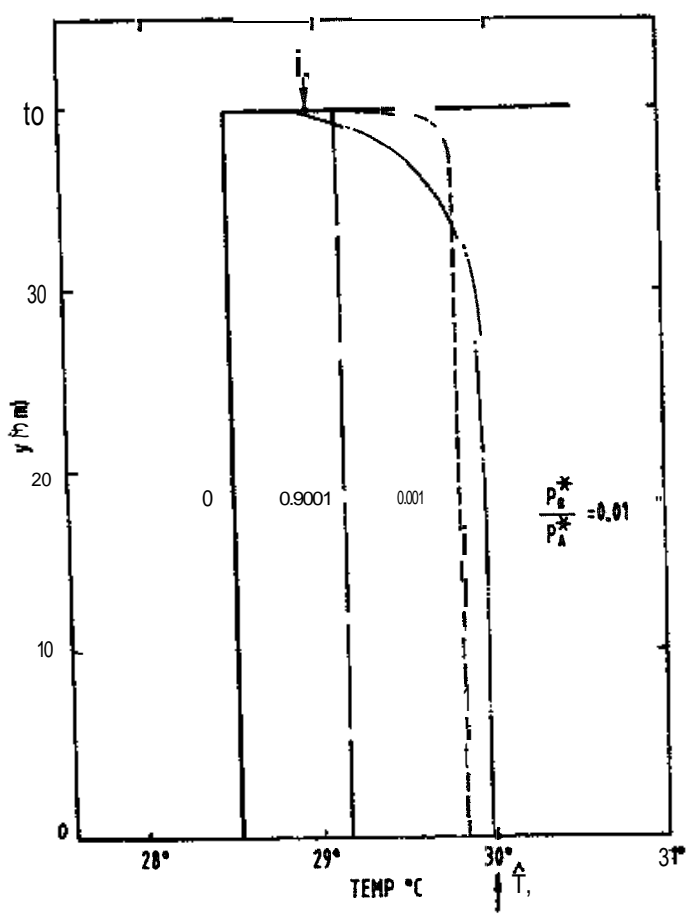

FIG. 4. Temperature distribution in water vapor. $\hat{T}_{0}=30$ "C, $\widehat{T}_{1}=29^{\circ} \mathrm{C}$; $\gamma_{\mathrm{A}}=1.325, \quad k_{\mathrm{A}}=0.019 \quad \mathrm{~W} / \mathrm{m}^{\circ} \mathrm{C} . \quad\left(D_{\mathrm{AB}}\right)_{1 \mathrm{~atm}}=0.000028 \mathrm{~m}^{2} /$ $\sec ; \lambda=1.0$.

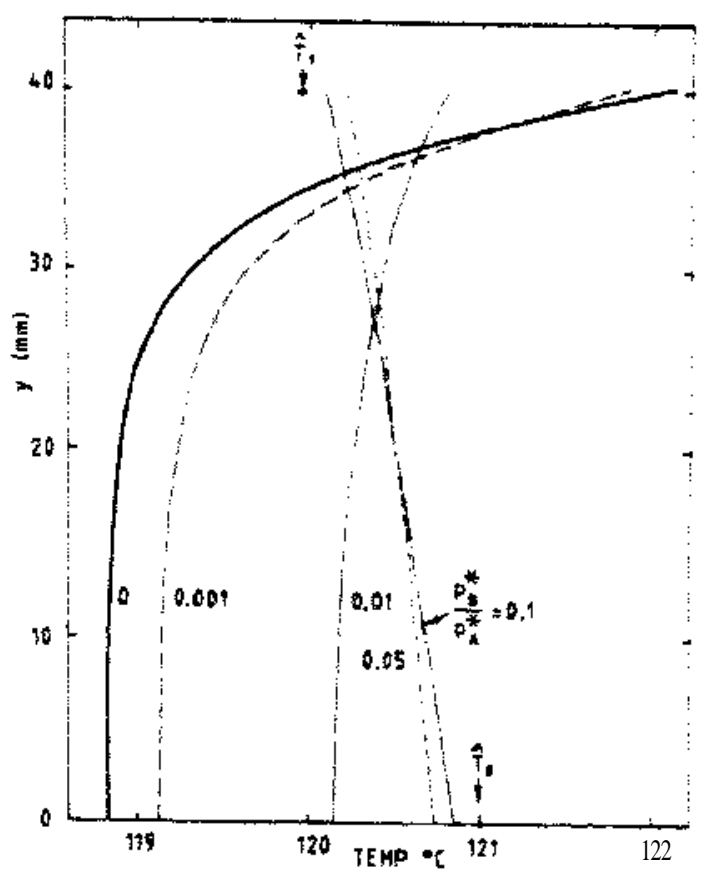

FIG, 5. Temperature distribution th mercurs vapor, $\dot{T}_{11}=121^{\circ} \mathrm{C}$. $T_{1}=120^{\circ} \mathrm{C}: \gamma_{\mathrm{A}}=1.67, \quad k_{\mathrm{a}}=0.00772 \mathrm{~W} / \mathrm{m}^{\circ} \mathrm{C}, \quad D_{k}$ $=0.00001124 \mathrm{~m}^{2} / \mathrm{sec}, \dot{A}=1.0$.

(3) For a given $\Delta \hat{T}$ the temperature jump is large if $K_{2}(1+\beta)$ is large, We must choose fluids with large $(1+P)$ at laboratory temperatures: this implies fluids with high boiling points. As regards $K$, it is obvious that $\gamma$ has to be kept large and the contamination low, In this connection, note that in (15)

$$
\begin{aligned}
\frac{2 H}{\rho^{*} D_{\mathrm{AB}}} \frac{p_{\mathrm{B}}^{*}}{\left(2 \pi R_{\mathrm{A}} T^{*}\right)^{t / 2}} & =\frac{R T^{*}}{p^{*}} \frac{2 H}{D_{\mathrm{AB}}} \frac{p_{\mathrm{B}}^{*}}{\left(2 \pi R_{\mathrm{A}} T^{*}\right)^{1 / 2}} \\
& \approx \frac{H\left(2 \pi R_{\mathrm{A}} T^{*}\right)^{1 / 2}}{\pi\left(p^{*} D_{\mathrm{AB}}\right)}-p_{\mathrm{E}}^{*} .
\end{aligned}
$$

Since the diffusion coefficient is approximately inversely proportional to the pressure, the implication is that it is the absolute value of $p_{\mathrm{B}}^{*}$, not the percentage contamination, that is important. Thus one can safely work at low total pressures. The height $H$ should be kept small and a low gas constant $R_{\mathrm{A}}$, i.e., large $M_{\mathrm{A}}$, should be beneficial,

(4) The points above suggest that we work with a high molecular weight vapor, but one made up of monatomic or diatomic molecules. Note that a high $\boldsymbol{M}_{\mathrm{A}}$ will also mean a low $C_{P A}$, which will help to increase $\delta$. Water isby and large an unsuitable fluid. Apart from its low molecular weight and high vapor pressure at room temperature it has thigh latent heat. For the pure vapor calculation shown in Fig. 4 the power requirement is about $4.8 \mathrm{~kW}$. If one is to work with water it should be at low temperatures; this is an intriguing possibility.

\section{III.APPARATUS}

An overall schematic of the experimental setup is shown in Fig. $\dot{6}$. The phase change phenomenon takes place in a 


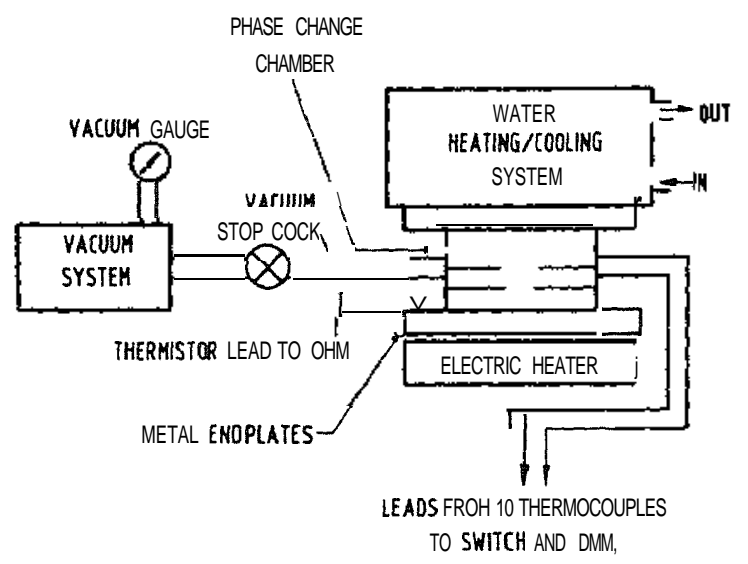

FIG. 6. Overall schematic of the experimental setup.

cylindrical chamber bounded by metal endplates. The chamber, which has thermocouples epoxied into it, is connected by a special rubber vacuum hose to the vacuum system through a vacuum isolation stop cock. The bottom endplate of the chamber is heated electrically while the top plate can be heated or cooled by a flowing water system. The leads from the thermocouples go through a rotary switch to a digital microvoltmeter. The temperature of the bottom plate is monitored by a thermistor. We observe that this basic setup could be (and was) modified depending on the fluid or the objective, to relocate the vacuum gauges, stop cocks, etc.

Most of the experiments reported here were carried out in Perspex (acrylic) chambers whose inner diameters were $105 \mathrm{~mm}$. Perspex was chosen because of its easy machinability, its transparency, poor thermal conductivity, and availability. We were aware that it was inferior to glass when we consider degassing, proneness to attack by organic fluids, high temperature properties, etc. However, the comparative ease of inserting a fairly large number of thermocouples was a major positive factor. Table II lists the Perspex chambers used, their dimensions, and special characteristics. Here, $t$ is the wall thickness, $H *$ is the height of the chamber, and $\bar{H}$ is the distance between the pedestals on the endplates. Note that the actual height between the liquid surfaces, $H$, is less than $\vec{H}$. Initial experiments quickly established that the flows in the chambers were almost one dimensional even for the largest $\bar{H} / D$ used (i.e., about 0.41 ). The theory in Shan$\operatorname{kar}^{2}$ shows that the principal reason for using small $H / D$ is to reduce the effects of contamination. We note that the three later chambers P1, AN 1, and AN2 each had a separate inlet to introduce the liquid; this inlet could also be used to

TABLE II. The Perspex chambers used. All dimensions are in millimeters.

\begin{tabular}{cccccccc}
\hline Designation & i.d. & $\boldsymbol{t}$ & $\boldsymbol{H}^{*}$ & $\bar{H}$ & $\begin{array}{c}\text { Flanged? Liquid } \\
\text { inlet? }\end{array}$ \\
\hline$A$ & 105 & 4.5 & 35 & 34 & Yes & No \\
$B$ & 105 & 4.5 & 50 & 49 or 43 & Yes & No \\
P1 & 105 & 4.5 & 50 & 40 & No & Yes \\
AN1 & 105 & 15 & 50 & 43 & No & Yes \\
AN2 & 105 & 15 & 25 & 18 & No & Yes \\
\hline
\end{tabular}

monitor the pressure in the chamber. Of course, all the models had an outlet to be connected to the vacuum system. Sealing between the chambers and their endplates was achieved using standard "O rings."

A closed quartz chamber was also fabricated to try to avoid the problem of degassing from the Perspex chamber walls and to improve the temperature and vacuum capabilities. This chamber had a single outlet for the vacuum connection and the thermocouples had once again to be epoxied into the chamber. The chamber had an inside diameter (i.d.) of around $100 \mathrm{~mm}$ and an internal height of about $32 \mathrm{~mm}$.

Every chamber had epoxied into it ten thermocouples to measure the temperature. The thermocouples were 32 gauge ( $<0.3 \mathrm{~mm}$ diam) Chromel-Alumel pairs with the welded junction about $40 \mathrm{~mm}$ from the chamber wall. The thermocouples were equally spaced to be $36^{\circ}$ apart (seeFig. 7) while their heights varied from close to the bottom plate to close to the top plate. Thus the thermocouple beads lie along a spiral near the center of the chamber and are therefore capable of indicating the temperature distribution in it. In order to eliminate spurious junction effects, wires of similar metals were used to connect the switch, copper thermal block, and digital voltmeter (DVM). Tests were carried out by heating various external junctions to ensure that the primary signals were not corrupted.

All measurements of voltage and resistance were carried out using a Keithley model 192 digital multimeter. This instrument has excellent stability and minimal drift. Many experiments with the setup convinced us that our voltage readings could be relied upon to be within $+2 \mu \mathrm{V}$ of the actual value; for Chromel-Alumel thermocouples this corresponds to $\pm 0.05^{\circ} \mathrm{C}$.

The vacuum system consists of a rotary pump capable of bringing the pressure in the pump chamber to less than 0.001 Torrwith a dummy on the inlet; the diffusion pump can then bring the pressure in the pump chamber to less than $5 \times 10^{-5}$ Torr. Naturally, the vacuum achievable in our ex-
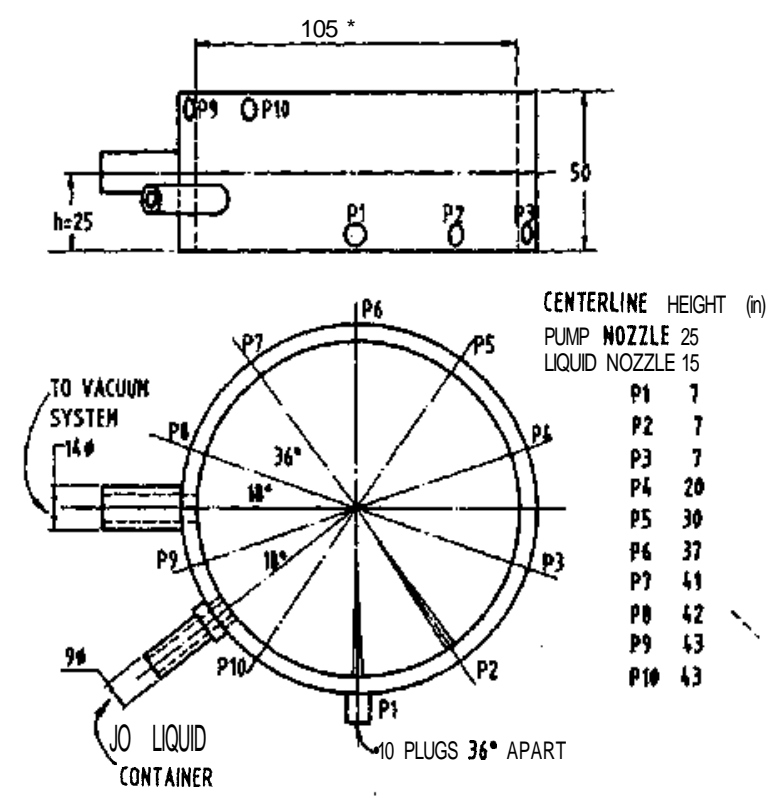

FIG. 7. Layout of thermocouples inside the chamber $\mathbf{P} 1$. 
perimental chambers could not be as high; e.g., with chamber AN2, in which some of the mercury experiments were carried out, the vacuum achievable was around 0.001 Torr.

The heating of the bottom plate was achieved by the use of an electric heating coil. The lower plate temperature was monitored using a thermistor. The upper plate could be cooled or heated as need be by the use of a flowing water system. We note here that the quartz chamber had quartz endplates fused to the cylindrical portion. In order to improve thermal contact thin beds of fine aluminum powder between the quartz endplates and the heating and cooling systems were used.

More details of the apparatus can be found in Shankar and Deshpande ${ }^{11}$ and Deshpande and Shankar. ${ }^{12}$

\section{EXPERIMENTAL PROCEDURES}

Prior to the actual experiments with evaporating fluids, tests were done to establish the accuracy and stability of the measuring systems. The raggedness, consistency, and repeatability of the thermocouples was most encouraging.

For every chamber, tests were first done with air in the chamber. The heights of the thermocouple junctions above the pedestal on the bottom plate were carefully measured with a metal rule or a cathetometer. Temperature distributions in air were then measured at high and low pressures to see whether normally expected Benard cell (laminar and turbulent) and classical heat conduction modes could be observed. These results proved quite satisfactory. ${ }^{\text {" }}$

For the tests with fluids the procedures were slightly different depending on whether the vapor could be allowed to contaminate the diffusion pump oil or not. For vapors like water vapor and Freon which adversely affect the diffusion pump oil, the liquid was injected into the chamber after evacuation was complete. For mercury the evacuation of the chamber was done after the liquid was inserted into the chamber.

A test was then conducted as follows. The heights of the thermocouple junctions were carefully measured and the chamber was then sealed and evacuated. Once the highest achievable vacuum was reached and held for some time, a temperature measurement (in the contaminant air) was taken. The liquid was then carefully poured avoiding air bubbles. After the measurements in air were completed, the chamber was isolated from the pump and the liquid let in under gravity. A series of temperature measurements was then taken sweeping from the lowest thermocouple to the highest and down again and so on. Since the evaporation process is such a rapid one, we obtain in this way a series of quasisteady temperature profiles as the system adjusts to the unsteady temperature conditions at the endplates and to the increasing contamination through leakage.

In the case of mercury, the evacuation was done with the mercury in place in the chamber. The results therefore correspond much closer to steady-state conditions except for the contamination from leakage. Special precautions had to be taken with mercury to prevent it from attacking parts of the apparatus. For example,the steel plates had to be nickel-plated. The thermocouples that were in the liquid had theirjunc- tions coated with insulating epoxy. Theother thermocouples were unprotected as tests

they were unaffected, at least over the time ofour experiment. It may be noted that freshly distilled mercury was used at the start of a ran with mercury,

\section{¥. EXPERIMENTAL RESULTS}

As was stated earlier, a series of temperature measurements in air were made over a range of pressures from around $10^{-2}$ to 760 Torr and a range of temperature differences from $8{ }^{\circ} \mathrm{C}$ to $20{ }^{\circ} \mathrm{C}$. This corresponded to a range of Rayleigh numbers Ra from $10^{- \text {fe }}$ to $9 \times 10^{4}$. It is known for the setup under consideration that
$\mathrm{Ra}<1700$
leads to pure conduction.
$1700<\mathrm{Ra}<47$ (XX)
to Benard cell structure,
$\mathrm{Ra}>47000$
leads to irregular turbulence.

The measured profiles conformed well with these known results.

Having established the capability of our measuring system with the air data we obtained data from the phase change experiments. In all the figures that follow a thermocouple output of $39.8 \mu \mathrm{V}$ corresponds to $1{ }^{\circ} \mathrm{C}$, with all temperatures measured with respect to the thermal block temperature. In Fig, 8, which shows data acquired with water, the initial profile in air prior to water injection shows good linearity corresponding to the pure conduction case; the pressure of the air was estimated to be around 1-2 Torr. The temperature profiles after the injection of water are much steeper and with time slope increasingly to the left. The profiles can be understood as follows. The injected water cools the lower plate by conduction and evaporation and the condensing water vapor heats the upper pkte. The high mass flux causes a quasisteady state to be established very rapidly; the high mass flux also accounts for the steep proile [see Eqs. (2) and (4)]. The vapor pressure relevant to this case is around 30 Torr and the contamination level is around 5\%. Theoretical calculations ${ }^{2}$ show that with this level of conta-

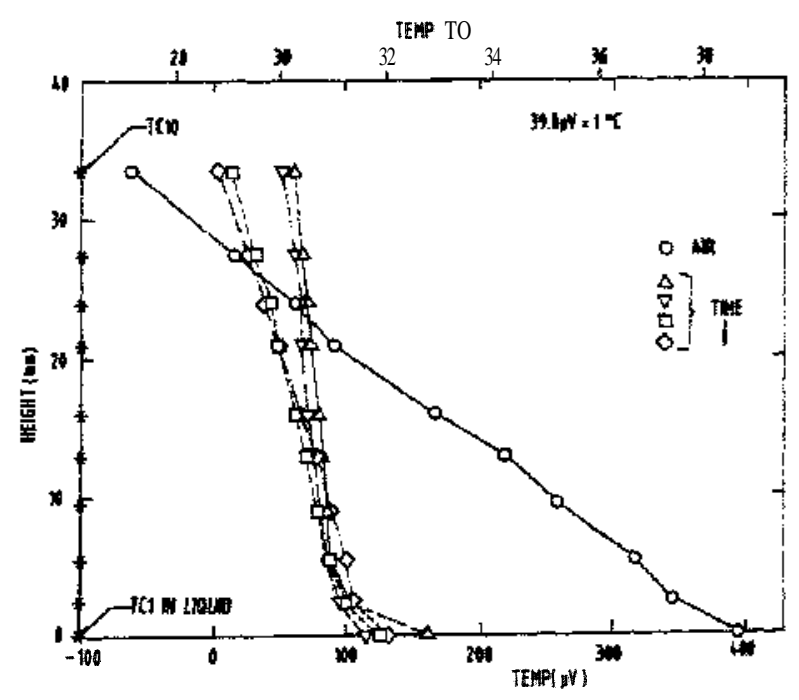

FIG. 8. Typical results for experiments with water. Model A. 
mination (with air) the temperature profile is expected to be normal with very small temperature jumps; thus the observed profiles conform qualitatively to what one would expect for water vapor contaminated with air.

We wish to point out an important fact regarding the profiles shown in Fig. 8. The experimental procedure adopted does not permit us to select in advance and maintain a given temperature differencebetween the hot and cold water films. The temperatures of the hot and cold aluminum plates are selected prior to water injection (e.g., approximately 394 $\mu \mathrm{V}$ and $-64 \mu \mathrm{Vin}$ this case). The subsequent water film temperatures and quasisteady vapor temperature profiles are determined by among other factors, the thermal inertia of the metal plates, the thickness of the films, the level of contamination, etc. Initially the bottom plate is cooled by the evaporating fluid and the upper plate is heated by the condensing vapor. Subsequently, the tendency willbe for the bottom plate and hot film to heat up due to the heat source and for the top plate and the top film to cool due to the heat sink. Both these effects can be seen in Fig. 8 from the data pertaining to TC's (thermocouples) 1 and 10.

Data typical of the results obtained from injecting Freon 113 around room temperature $\left(30^{\circ} \mathrm{C}\right)$ are shown in Fig. 9. Again, prior to the injection of the fluid the air temperature profile (at a pressure of 1-2 Torr) is quite linear; TC's 8 and 9 read high because they were bent upward to have their beads close to the upper plate. After the injection of the fluid the profiles are almost vertical indicating a rather large evaporating mass flux. As opposed to the water case, all five curves show a cooling of the whole field with time; the lower plate had not begun to heat up again during the course of the readings. The slight differences in slope between adjacent curves may also be due, at least partially, to the time taken for measurement by alternate upward and downward sweeps as the system was cooling. The contamination in this case was around $0.5 \%$ since the vapor pressure ofFreon 113 at these temperatures is around 420 Torr. Theoretical calculations indicate ${ }^{2}$ that for this fluid, whose $y$ value is around 1.05 , the temperature jumps will be small irrespective of the purity of the vapor. Thus the curves of Fig. 9 conform qual-

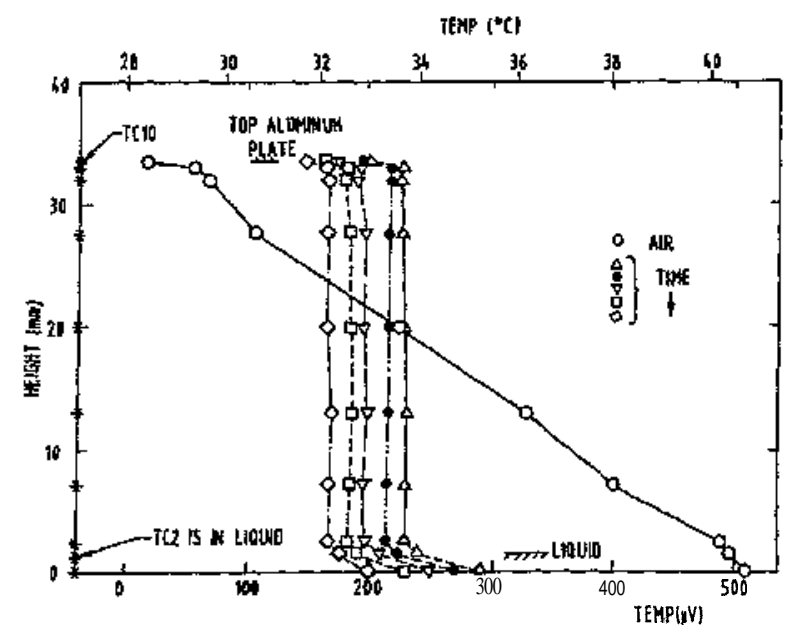

FIG. 9. Typical results for experiments with Freon 113. Model A. itatively to the predictions of the theory for the instance where the mass flux is high.

An advantage of having the chambers made of a transparent material was that certain features of the phase change phenomenon could be seen. For example, the formation of liquid films on the upper plate, the flow of liquid down the sides of the chamber, the formation of waves on the lower water surface by the impact of falling drops, the position of TC 2 with respect to the liquid surface, etc., could all be observed. A small detail might be pointed out: the pedestals on the aluminum endplates had to be roughened with emery paper in order for proper liquid films to form on them. Smooth machined surfaces are not sufficiently wettable.

The analysis in Sec. II clearly points out the great benefits of working with a vapor having high $y$ and high molecular weight. It was for this reason that we chose to work with mercury in spite of its poisonous and corrosive nature. All the results shown in Fig. 10, with mercury as the working fluid, were obtained from chamber AN2 (see Table II). This thick-walled chamber, fabricated after much earlier experience, had superior vacuum capabilities; in the absence of mercury it was possible to achieve a vacuum of around 0.001 Torr. As indicated earlier, in these experiments the evacuation was done with the mercury in situ; thus these results correspond much closer to "steady-state" conditions. We also note that TC's 1,2 , and 3 were used to measure the temperature in the hot liquid while TC 10 measured the upper cold film temperature; the former were coated with epoxy to resist corrosion by hot mercury and for electrical insulation purposes.

The data shown in Fig. 10 represent just a small sample of data obtained with mercury over a large range of temperatures. Since in Fig. 10(a) the thermal block temperature was $29.5{ }^{\circ} \mathrm{C}$ corresponding to $0 \mu \mathrm{V}$, the hot and cold liquid mercury temperatures are around $60^{\circ} \mathrm{C}$ and $24^{\circ} \mathrm{C}$, respectively. In Fig. 10 (b) the corresponding temperatures are $30.5^{\circ} \mathrm{C}$ for the thermal block and $100{ }^{\circ} \mathrm{C}$ and $22^{\circ} \mathrm{C}$ for the hot and cold mercury liquid temperatures. Though some drift is observable in the data of Fig. 10(a), because of drift in the boundary conditions, the large jumps at the interfaces are clearly visible. The temperature jumps near the boundaries are even more dramatic in Fig. 10(b), where data were obtained after more than $10 \mathrm{~h}$ of continuous evacuation, and with better control on the cold side temperature. We should point out, however, that in this run TC's 4 and 8 were found to be making contact with the lower and upper liquid surfaces, respectively; hence data from these locations were not available. Nevertheless, the remaining data clearly establish large jumps near the boundaries, close to $50 \%$ of the applied temperature difference. Note that TC 9 is within $0.8 \mathrm{~mm}$ of the top plate.

The mercury data reported above were obtained after moderate to long periods of evacuation in order to eliminate the contaminant air. In these experiments the vacuum stop cock between the experimental chamber and the vacuum system had been removed to reduce the pressure drop and so the data were obtained while the pump was still connected to the chamber. Figure 10(c) shows that this procedure is unlikely to cause serious errors. The circles in Fig. 10(c) were 

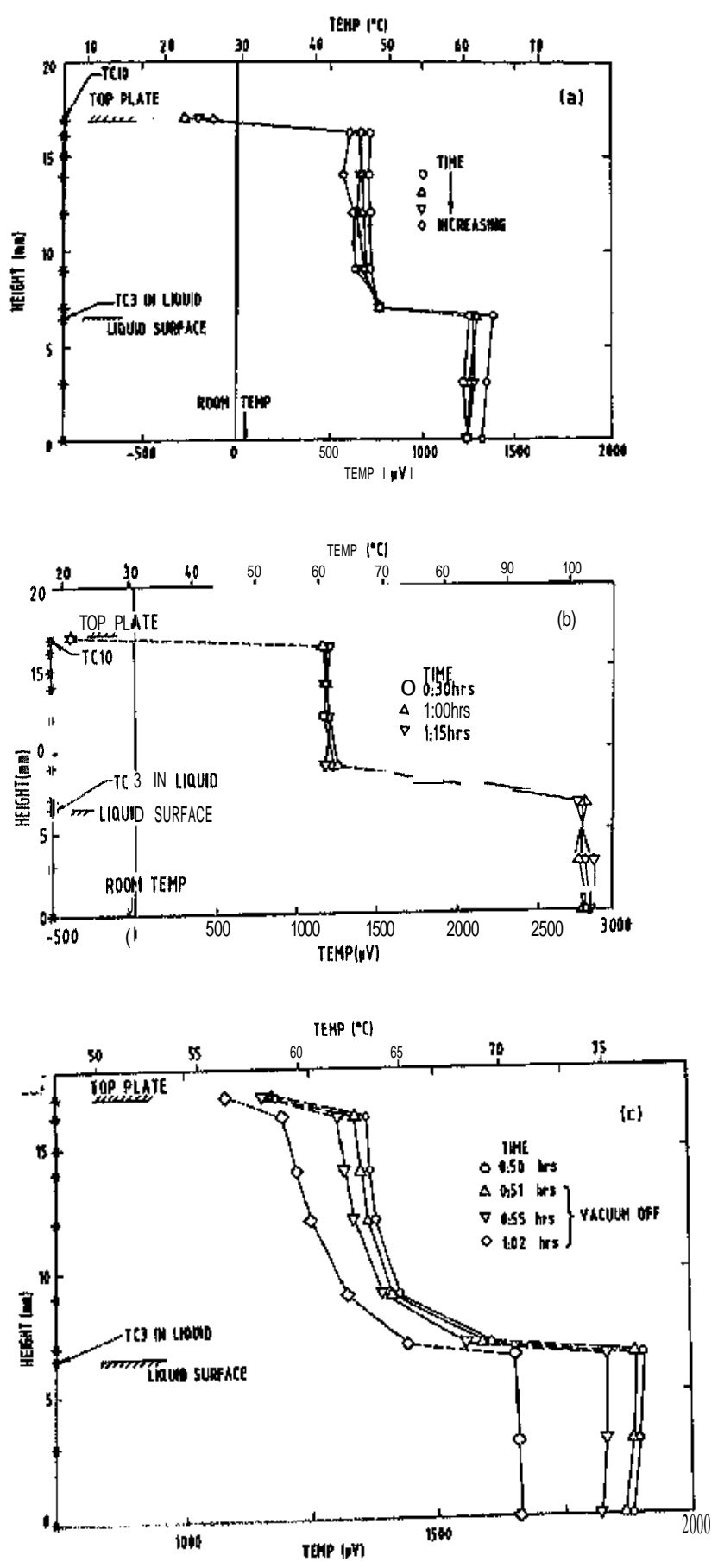

IIGi. 10.(a) Temperature profiles in mercury vapor. Saturation vapor pressure at lower liquid temperature, $\hat{p}_{0} \approx 0.025$ Torr, and corresponding meanfrec path $l_{12} \approx 1.6 \mathrm{~mm}$. (b) Temperature profiles in mercury vapor; $\hat{p}_{0}=0,27$ Forr, $I_{0}=0.17 \mathrm{~mm}$. (e) Effects of switching off vacuum and increasing contamination; $\hat{p}_{01}: 0.07$ Torr, $l_{0}=0.6 \mathrm{~mm}$.

obtained with the pump still on after a period of evacuation. The rest of the data on Fig. 10(c) were obtained with the pump isolation valve activated, i.e., with the pump off. Notice that the later data are qualitatively very similar to the data with the pump on, and merge into it. The principal effect is the gradual lessening of the steepness of the curves caused by the increasing contamination due to the air leaking in.

In Shankar and Deshpande ${ }^{1.3}$ we present data for $\hat{T}_{0}$ ranging from $28{ }^{\circ} \mathrm{C}$ to $100{ }^{\circ} \mathrm{C}$, the upper limit for chamber
AN2. In this range the mean-free path $\hat{l}_{0}$ varied from around $16 \mathrm{~mm}$ to $0.17 \mathrm{~mm}$. Since the gap width $H$ in our apparatus was around $10.5 \mathrm{~mm}$ the flows ranged fromfree molecular to near continuum, if not continuum, conditions. In all these cases the jumps are clearly visible and, if anything, more dramatic at the high pressure limit. A related issue is that of the response of the thermocouples at these low densities. The possible errors resulting from this effect have been discussed in Shankar and Deshpande ${ }^{11}$ where it was concluded that the relative temperatures in all probability are not affected much. In any case, our experience has so far convinced us that these simple and rugged devices are reliable for the present purposes.

The temperature profiles in Fig. 11 were obtained from the quartz chamber. As mentioned before, this closed chamber was designed to conduct the experiment at elevated temperatures, to obtain better vacuum, and to avoid degassing from the Perspex. ${ }^{12}$ Since the chamber was closed the thermocouple positions were fixed once and for all. All the TC's were coated with epoxy for $5 \mathrm{~mm}$ at the root near the chamber wall and TC's 1 and 2 were fully coated to protect them from hot mercury and to insulate them electrically. The tips of TC's 10 and 11, which entered the chamber from the same hole, were coated forming small beads that were in contact with the top quartz wall.

Experiments were conducted with hot mercury temperatures ranging from $90^{\circ} \mathrm{C}$ to $140^{\circ} \mathrm{C}$. It was not possible to satisfactorily measure the temperature of the top mercury film since the protective beads on TC's 10 and 11 had only a small contact area with the top plate and hence measured a temperature that was in between the top film temperature $\widehat{T}_{1}$ and the vapor temperature near the top plate $T_{1}$. It is for this reason that we are not able to measure the top temperature jump in this chamber. Note that this difficulty did not exist for the Perspex chambers. However, there was no difficulty in the measurement of the bottomjump as seen from Fig. 11. Thermocouples 1 and 2 measure different temperatures as before in the Perspex chamber AN2 and this may be due to the presence of Bénard cells in the mercury pool or due to nonuniform heating. The mercury surface temperature is assumed to be the average of these two values.

We wish to observe here that the temperature jumps obtained with the Perspex chambers were once again obtained over a much larger temperature range with $T_{0}$ as high

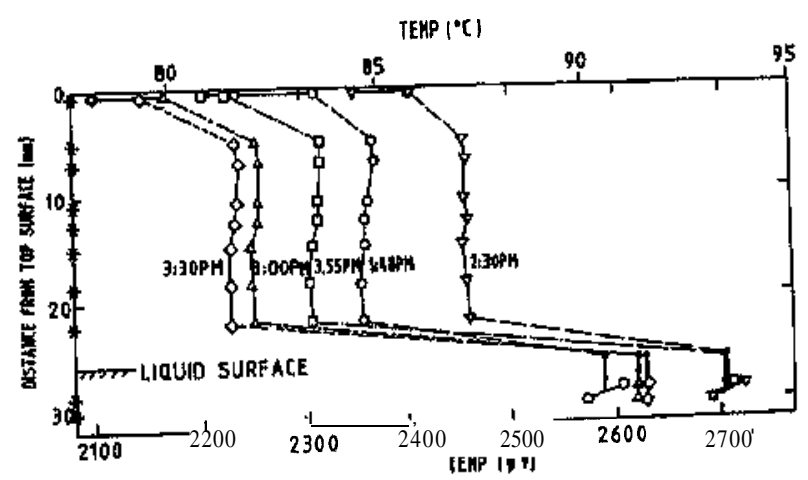

FIG. 11. Temperature profiles in mercury vapor obtained from the quartz chamber. 
as $140{ }^{\circ} \mathrm{C}$. This corresponds to a mean-free path $\hat{l}_{0}$ (based on $\widehat{T}_{0}$ ) as small as $0.03 \mathrm{~mm}$, thus extending the data further into the continuum regime. In Fig. 11 the curves translate in time because of our inability to control the boundary conditions inside the safety enclosure. We do not claim that the mild inversions observable in Fig. 11 are genuine anomalous profiles; they could be due to three-dimensional effects, nonuniform heating, etc. The jumps at the lower boundary are of course manifest; these are not so obvious at the upper boundary because of our inability to measure the upper film temperatures for the reasons stated earlier.

It may be noted in Figs. 10 and 11 that the temperatures in the mercury pool are not uniform and often the upper TC's read higher than TC 1 . The most obvious explanation is nonuniform heating of the pool with azimuthal variations of temperature. But calculations established that a competing cause (for example, in Fig. 11) is the formation of Bénard cells in the liquid. Note, however, that these nonuniformities do not affect our conclusions regarding the large temperature jumps, in view of the magnitude of the latter.

The possible sources of errors and their implications have been discussed in Shankar and Deshpande. " Sources of a geometrical nature, such as lack of one-dimensionality in the apparatus, have been minimized by using small $H / D$, using chambers made of materials with poor thermal conductivity, etc. The experimental results, for example, the linear profiles in air in the conduction regime, uniform profiles with rapidly evaporating vapors, etc., seem to indicate that the geometrical factors are not too serious. Recall that the thermocouple tips lie on a spiral around the chamber. Among the nongeometrical sources of errors the principal one is due to heat conduction along the TC wires. This has been minimized by exposing a large fraction of each TC to the same nominal temperature in the quasi-one-dimensional temperature field. The choice of Chromel-Alumel TC's, with their low thermal conductivity, was also dictated by this consideration. Representative calculations show that the errors due to this source are likely to be small and since they are of the same sign and magnitude they are not likely to be of any consequence as far as relative temperature measurements are concerned.

\section{DISCUSSION AND CONCLUSIONS}

We have in this paper presented measurements of temperature profiles in liquid-vapor phase change between plane liquid surfaces. As we have emphasized earlier, the theory presented in Sec. II clearly indicates the need to work with a monatomic vapor like mercury and to reduce contamination to a minimum.

The issue of contamination and its estimation is a very complex one. While we are confident that the level of contamination by air was less in the quartz apparatus than in the Perspex chambers, no quantitative estimates could be made. Again, while degassing from the Perspex was avoided, degassing from the epoxy coatings could not be avoided especially at the higher temperatures. We also observed that especially over large run times the surface of the hot mercury pool tended to collect scum. Paradoxically while a large ran time might help to reduce the effect of degassing, the liquid surface on the other hand might become increasingly contaminated. Since the phenomena of interest are governed mainly by conditions near the surfaces we can also expect surface contamination to play an important role. Unfortunately, there are no models at present to estimate the effects of surface contamination.

The principal conclusions of this paper are the following.

(1) From the theoretical analysis it is important to work with monatomic vapors with high molecular weight and at fairly low pressures.

(2) Contamination, according to the analysis, has to be reduced to a minimum as its presence drastically reduces the temperature jumps at the interfaces.

(3) Large temperature jumps exist at the interfaces. These jumps exist for conditions ranging from very low densities to continuum conditions. Since we had no way of measuring contamination levels it was not possible to estimate the maximum possible jumps. With our present contamination levels we have observedjumps as large as almost $50 \%$ of the applied temperature difference. Hence the only issue that remains to be settled is that of the anomalous temperature distribution. To settle this matter one would probably require a better control on contamination and on the apparatus.

(4) The jumps exist even when the applied temperature differences are large, i.e., as large as $80^{\circ} \mathrm{C}$. One might therefore suspect that as far as theory is concerned the jumps are not a result of linearization.

(5) Continuum calculations which assume that the temperature jumps are absent, such as those of Plesset, ${ }^{8}$ are certainly in error.

\section{ACKNOWLEDGMENTS}

We would like to gratefully acknowledge all the help that we have received from so many of our colleagues at the National Aeronautical Laboratory (NAL), especially Dr. P. S. Gopalakrishnan and Dr. K. Bandhyopadhyay. We would also like to thank the Director, NAL, for supporting this investigation. A brief announcement of some of these results was made as a letter by Shankar and Deshpande. ${ }^{1.4}$

${ }^{1}$ Y. P. Pao, Phys. Fluids 14, 306 (1971).

2P. N.Shankar, NAL Tech, Memo.T'M FM 880.3, 1988.

'Y, Sone and Y. Onishi, J. Phys. Soc. Jph, 44, 1981 (1978).

"K. Aoki and C. Cercignani, Phys. Fluids 26, $116.3(198: 3)$.

"L. D. Koffmun, M. S. Plesset, and L. l.ees, Phys. Fluids 27, 876 (1984).

"L. F, J. Hermans and J, J. M. Beenakker, Phys. Fluids 29, 42.31 (1986).

${ }^{7}$ C. Cercignani, W, Fiszdom, and A. Fitezzolti, Phys. Fluids 28, 3237 (1985)

* M. S. Plesset, J. Chan. Phys. 20, 790 (1952).

"Y. Onishi, 14th Internaticmal Symposium on Rarefied Gas Dynamics, Tsukuba, Japan, 1984,

"IF. E. Marble, International Symposium on Fluid Dynamics of'teterogeneous and Multiphase Media, Institute of Acrodymamics, Naples, 1966 ,

"P. N. Shankar and M. D. Deshpande, NAL Tech. Memo. TM I'M 8804, 1988.

12 M, D. Deshpande and P, N. Shankar, NAL Tech. Memo, TM FM 8809, 1988.

${ }^{13}$ P. N, Shankar and M. D, Deshpande, NAL Tech, Memo, TM FM 8805, 1988.

${ }^{14}$ P. N. Shankar and M. D. Deshpande, Pramana 31, L337 (1988). 